\title{
Fighting Together: Discovering the Antecedents of Social Support and Helpful Discussion Threads in Online Support Forums for Cannabis Quitters
}

\author{
Kuang-Yuan Huang \\ Hasan School of Business \\ Colorado State University - Pueblo \\ kuangyuan.huang@csupueblo.edu
}

\author{
Yoanna Long \\ Hasan School of Business \\ Colorado State University - Pueblo \\ yoanna.long@ csupueblo.edu
}

\begin{abstract}
An increasing number of people are using online cannabis support forums as a source of help for their cannabis quit attempts. In order to assist support seekers dealing with emotional and physicalbehavioral difficulties associated with their cannabis abstinence, it is important to identify the factors that facilitate social support provisions by forum members, as well as the overall helpfulness of discussion threads. In this combined qualitative and quantitative study, we propose a model hypothesizing and testing these factors, based on variables generated using Natural Language Processing and Machine Learning techniques. The result shows that linguistic and content characteristics of thread-initiating messages are important predictors of the receptions of informational and emotional support from other forum members, and of the overall helpfulness of discussion threads.
\end{abstract}

\section{Introduction}

In recent years, the number of Americans supporting recreational marijuana use is rising. According to a 2017 survey conducted by the Pew Internet and American Life Project, 61\% of Americans believe marijuana use should be legalized, changing from 57\% in 2016 and nearly doubling what it was in 2000 [1]. With several states legalizing recreational marijuana, the number of marijuana users has been increasing [2], due to enhanced availability, greater social acceptance, and lower marijuana prices [3]. Similarly, more and more marijuana users are choosing to stop or reduce marijuana uses [4], attributable to some known physical, social, behavioral, mental adverse effects, and/or legal reasons $[5,6]$. Many of these people join online cannabis support forums looking for help with their cessation or reduction attempts, from like-minded others experiencing or have experienced similar situations [7]. A quick message count from the Reddit $\mathrm{r} /$ leaves forum, one of the largest online support group for cannabis quitters shows that the number of threads/messages posted to the r/leaves forum have gone up from 5,875 threads/36,935 messages in 2015 to 9,769 threads/65,920 messages in 2017.

One key reason behind people joining online social support forums in search for support from strangers is the social stigma associated with the health-related issues [8], which is the case for cannabis users [7]. The anonymity and invisibility nature of online social support forums makes forum users feel safe to disclose personal stories and exchange social support emotional support (support that restores emotional stability through the communication of love, sympathy, encouragement, etc.) and informational support (support that reduces uncertainty and/or facilitates problem-solving such as knowledge sharing) [8,9] with others having similar struggles. Despite the health promoting and personal empowerment effects of joining online social support forums $[8,10]$, and despite the finding that social support plays an important role for cannabis quitters to maintain their withdrawal attempts [11], little is known about the social and behavioral dynamics of online cannabis support forums. Specifically, little is known about what motivates the sharing of social support from forum users to help others, and little is known about what contributes to quality and helpful discussion threads discussion threads containing useful information or encouraging messages benefiting both support seekers and other forum members, including lurkers. Therefore, our study attempts to address these two research questions:

RQ1: What are the factors that drive members of an online cannabis support forum to provide informational and emotional support? 


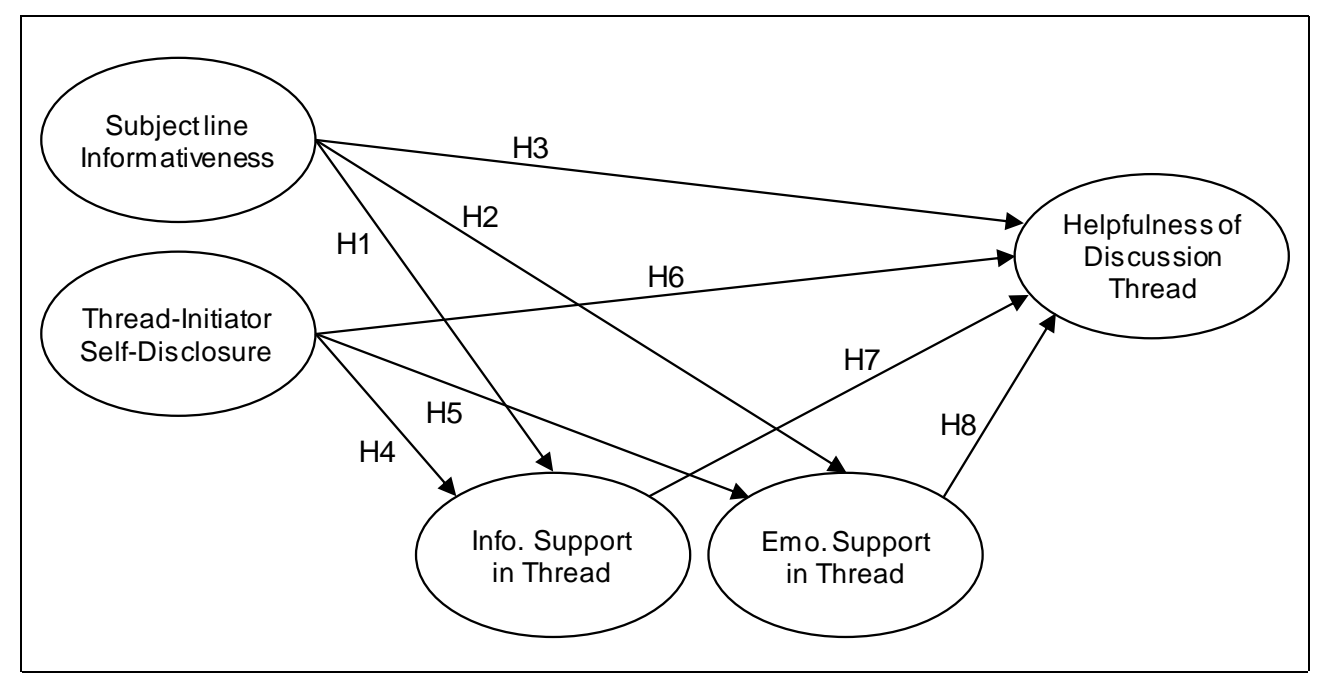

Figure 1. Research model

RQ2: What are the factors that lead to helpful discussion threads in an online cannabis support forum?

We believe that the social support and computational linguistics literature provides useful insights for answering the two research questions. We specifically propose a model hypothesizing and testing the effects of the linguistic and content features of subject lines of discussion threads and thread-initiating messages (i.e., the first message of each discussion thread) on discussion forum users' responses of informational and emotional support, and on the overall helpfulness of the thread. Additionally, we hypothesize that both informational and emotional support provided by forum users also contribute to helpful discussion threads.

This paper makes several research contributions. First, with the increasing number of cannabis users (and quitters), it is important to study different aspects of cannabis use behaviors, including behaviors related to cannabis withdrawal and relapse. Our paper contributes to this stream of study. Second, our study is among the first to study online cannabis support forums, leading to a better understanding of the social and behavioral dynamics of cannabis quitters in an online environment. Third, this study adopted various natural language processing and machine learning techniques to analyze a large volume of online messages in response to recent calls to adopt automated methods to study online user generated content (UGC) [12]. Our study provides practical implications for online cannabis support forum designers and users as well. For support seekers and providers of online cannabis support forums, this study provides suggestions on the ways of promoting helpful discussions benefiting forum users. For support forum designers and administrators, this study also provides useful insights into online community design and administration to facilitate supportive interactions.

The rest of the paper is organized as follows. Our proposed research model on the antecedents of social support provision and helpful discussions in online cannabis support forums and the associated hypotheses are presented first in the subsequent section, which is followed by the research method and results sections. A discussion of the findings and their research and practical implications are provided next. The paper ends with the limitations and conclusion section.

\section{Model and hypotheses}

Our proposed research model (Figure 1) takes into account linguistic and content features of threadinitiating messages and thread subject lines, and social support providers' behaviors - informational and emotional support provisions -, in online cannabis support forums. As discussed above, our purpose of the model development is to identify ways of promoting forum users' participations in discussion threads to provide social support, and to identify factors of facilitating helpful thread discussions. Studying linguistic and content features of thread-initiating messages and thread subject lines allows us to look into the psycho-social information about support seekers [13], and how this information affects support providers' behavior and the overall helpfulness of discussion threads.

\subsection{The impacts of thread subject lines on social support received and on thread helpfulness}


In order to have a helpful thread discussion for support seekers and other forum users, and to attract forum users to join a thread to provide social support, a well-crafted and informative thread subject line is critical. It has been well-documented in the literature, that in threaded discussions, thread subject line formulations can be strategic, that the information provided in thread subject lines can affect forum users' willingness to click into a thread [14], and that an informative and appealing subject line will lead to deeper and quality thread discussions [15].

First, the length of thread subject lines represents the amount and depth of information provided [16], which is especially crucial in the context of threaded discussions, where forum members decide whether to click into a given thread or not based on the subject line [15]. The amount of information provided in subject lines can help forum members understand the specific type of support needed by support seekers, determine whether or not they have experienced similar situations, decide if they have the information and knowledge to provide help, and/or whether they are interested in the discussion topic in general.

Additionally, information that is context-related in subject lines is more likely to attract forum users [15]. For cannabis quitters, due to shared experience, withdrawal symptoms experienced by support seekers - either emotionally-related (e.g., depression, nervousness, or angry outbursts) or physicallybehaviorally-related (e.g., hot flashes, lack of appetite, or headaches) [17] -, can especially remind other forum members of similar situations they had been through, leading to an increased empathetic discussion and the exchange of useful information, resulting in a helpful thread. Therefore, we expect that the discussion thread subject line informativeness - both the length of subject lines and the appearances of cannabis withdrawal symptoms (negative emotions and/or physically-behaviorally-related symptoms) - to be positively associated with social support responded by forum members, as well as with the overall helpfulness of the discussion thread. Based on the above discussion, we hypothesize that:

H1: Discussion thread subject line informativeness has a positive effect on the amount of informational support received in the thread.

H2: Discussion thread subject line informativeness has a positive effect on the amount of emotional support received in the thread.

H3: Discussion thread subject line informativeness has a positive effect on the overall helpfulness of the thread.

\subsection{The impacts of thread-initiating messages on social support received and on thread helpfulness}

Similarly, the amount of information provided in thread-initiating messages is positively associated with social support provided by forum members as well as thread helpfulness. Specifically, we posit that thread initiators' levels of self-disclosure in thread-initiating messages would convey useful information, attracting forum members to provide social support. Selfdisclosure is a primary support-soliciting strategy in online cannabis support forums [7]. In the online context, self-disclosure is positively linked to relationship intimacy [18]. This phenomenon occurs in online environments because, with the absence of nonverbal cues, message content is the sole information source for other forum users to know about support seekers. Therefore, the more support seekers disclose sensitive and personal information about themselves, the more familiar and intimate other forum users will feel they are with the support seeker. This leads to increased social support responses [19]. The increased self-disclosure and support exchange is especially the case in online social support forums where people tend to disclose more about themselves with an expectation to receive social support, which intensifies forum users' perceptions of relationship intimacy [8].

It has also been found that in online support forums, continued and helpful supportive interactions relies heavily on thread participants' disclosure of sensitive experience about themselves, as it helps support providers know more about support seekers' concerns, symptoms, emotional states, and so forth, thus knowing how to appropriately provide support to deal with support seekers' current difficulties [8, 20]. A better understanding from support providers about support seekers' various information and situations can also lead to more emphatic and helpful communications of support [21]. Based on the above discussion, we hypothesize that:

H4: Self-disclosure in a thread-initiation message is positively associated with the amount of informational support received in the thread.

H5: Self-disclosure in a thread-initiation message is positively associated with the amount of emotional support received in the thread.

H6: Self-disclosure in a thread-initiation message is positively associated with the overall helpfulness of the thread. 


\subsection{Associations between social support and the helpfulness of discussion threads}

Social support is about the "exchange of verbal as well as nonverbal messages in order to communicate emotional and informational messages that reduce the [support] retriever's stress" $[9$, p. 124]. It is interpersonal transactions that are performed to offer different supportive functions, and in the online context, two key supportive functions have been documented: 1. helping problem-solving and uncertainty reduction (i.e., informational support), and 2. facilitating emotional recovery (i.e., emotional support) [9]. People facing stressful situations join online support forum looking for support from likeminded individuals who "have been there" and discuss their fears, problems encountered, and exchange common experiences [8]. Many users of online support forums found that support received online can be more helpful than support received from healthcare professionals, especially those who feel stigmatized by their conditions [8], such as cannabis users [7]. Therefore, the more social support a support seeker receives from other online support forum users in a discussion thread, the more likely s/he find the thread helpful.

Social support provided in online support forum not only benefit support seekers alone, it helps other forum users as well, including lurkers who browse and read but rarely post messages [22]. This is because messages posted to online support forums are open to all forum users. Those who click into a thread, through browsing or searching, are likely to be people facing similar difficulties as the thread initiator and find the thread subject relevant and helpful. Therefore, support provided in a thread can benefit both thread participants and non-participants. Based on the above discussion, we hypothesize that:

H7: Informational support provided in a discussion thread has a positive effect on the overall helpfulness of the thread.

H8: Emotional support provided in a discussion thread has a positive effect on the overall helpfulness of the thread.

\section{Research method}

To test the proposed research model, we collected and analyzed the content of messages posted on a large online cannabis support forum. Natural Language Processing and Machine Learning approaches were adopted to analyze online message content automatically and generate variables for this study. By using variables generated from the actual message content posted by users of the target support forum, we were able to acquire insights into the values, beliefs, attitudes, intentions, and behaviors of message posters [23].

The target online cannabis support forum is the Reddit $\mathrm{r} /$ leaves community, one of the largest online support community for individuals quitting cannabis uses, with about 64,500 forum members as of May 2018. The mission of the support forum, as is put on the forum main page, is:

This is a support and recovery community for practical discussions about how to quit pot, weed, cannabis, edibles, BHO, shatter, or whatever THC-related product you're using, and support in staying stopped.

From Jan. to Apr. 2018 there were 5,762 threads initiated, with an average of 337 messages posted per day. For the purpose of this study, we downloaded discussion threads initiated between Jan. 2015 and Dec. 2016, resulting in 13,770 discussion threads. After removing deleted threads, there were 12,675 threads remained, containing 83,009 messages $(29,132$ of the messages were those made by initiators of those threads), for testing our proposed model. The average number of messages per thread was 6.5 , and of which a support seeker/thread initiator on average made 2.3 postings. An example of a thread-initiating message is listed below (the following messages were paraphrased to protect author identity):

[Subject] How can I quit the weed?

[Content] It is extremely hard to do so alone. Anything I've done be it positive or negative I've done while blazed. I am wondering if I've become too dependent on it. I've been smoking for about 6yrs. I think I am ready to quit.

And, this is an example of social support messages provided by forum members as a response:

[Content] Cut all contact with your dealers, delete numbers, and so on. You need to be concrete with yourself and say, this is it. It's all in your head. You can certainly quit if you really want to. If you pick up weed again, you're just being weak and chasing the high.

Based on the collected data, our goal was to see if and to what extent, for each discussion thread, the linguistic and content features of the thread subject line and thread-initiating message would affect social support posted in the thread as well as the overall thread helpfulness. We also wanted to examine if 
social support messages posted by support providers would predict the helpfulness of a thread.

\subsection{Measures}

3.1.1 Independent variables. To generate independent variables, we used the Linguistic Inquiry and Word Count (LIWC) software package [13] to analyze online message content and subject lines. LIWC is a research tool used to analyze text documents and count the frequencies of the occurrence of words belonging to each of the 73 pre-defined word categories including pronouns (e.g., I, We, She), negative emotion (e.g., afraid, agony, nervous), biological process (e.g., abdomen, muscle, sleep), and so on. To prepare for content analysis using LIWC to generate our independent variables, we extracted, from collected discussion threads, $(12,675)$ thread subject lines and thread-initiating messages.

Thread subject line informativeness: Three formative indicators were generated to measure subject line informativeness. First, we counted the number of words in a given thread subject line as an indicator for this model construct. Next, we examined the subject lines to see if cannabis withdrawal symptoms appeared in them, resulting in two indicators - negative emotion in subject line and physical-behavioral symptoms in subject line. The LIWC "negative emotion" word category was used to identify negative emotion words used by the initiator of a given thread in the subject line. As indicated above, negative emotion is one key withdrawal symptoms experienced by cannabis quitters [17]. We coded the "negative emotion in subject line" variable as " 1 " if any negative emotion word appeared in the subject line, otherwise as " 0 ."

The LIWC "biological process" word category was used to identify words related to physical-behavioral symptoms used by the initiator of a given thread in the subject line. This word category contains four subcategories - body, health, sexual, and ingest -, through which word indicating physical-behavioral symptoms of cannabis withdrawal could be captured. We coded the "physical-behavioral symptom in subject line" variable as " 1 " if any biological process word appeared in the subject line, otherwise as " 0 ."

Self-disclosure in thread-initiating message: To capture thread initiators' levels of self-disclosure in their thread-initiating messages, two reflective variables were generated. First, we followed previous studies [24] and used the LIWC categories including 1st-person singular pronoun, 1st-person plural pronoun, social (including subcategories: family, friend, female, and male), perceptual processes (including subcategories: see, hear, and feel), positive emotion, and negative emotion, to identify self- disclosure words in thread-initiating messages. For each discussion thread, this variable is generated by counting the number of occurrences of self-disclosure words in the thread-initiating message.

We used the diversity of topical themes mentioned in thread-initiating messages as the second reflective indicator of the self-disclosure construct. As indicated above, knowledge about different aspects of a support seeker will lead to more emphatic and helpful communications of support [21]. To measure the levels of topical diversity of thread-initiating messages, we followed [25] and used the Latent Dirichlet Allocation (LDA) topic modeling approach [26] to automatically identify discussion themes covered across all the 12,675 thread-initiating messages in the collected data. By analyzing word use frequencies and occurrences in texts, LDA, a machine learning technique, is able to infer topical themes (i.e., word-use patterns) that characterize the document collection [26]. Seven main topical themes were identified automatically based on the collected thread-initiating messages. An example of the identified topical themes is about sleep difficulties, characterized by word uses such as dream, hour, nightmare, sleep, and sweat. Another example of the topical themes identified is related to daily social lives, containing keywords such as friend, pot, school, home, and job. Based on the discovered topical themes, we then calculated the topical distributions of threadinitiating messages across the seven themes. The higher the extent to which a given message covers different topical themes, the more diversified personal information was disclosed in the message, covering different aspects of personal withdrawal experience, story, and/or history. This resulted in a value range between 0 (least topical-diverse) to 1 (most topicaldiverse).

\subsubsection{Dependent variables.}

Informational and emotional support received from forum users: Followed the automated social support classification procedure discussed in [27], we applied machine learning techniques to classify thread responses (excluding messages posted by thread initiators) into either emotional support or informational support. First, from the collected 53,877 thread responses posted by forum members a random of 600 messages were selected and classified manually into either support type. ${ }^{1}$ These manually classified messages were then used to "train" the machinelearning program incorporated with the Support Vector

\footnotetext{
${ }^{1}$ The unit of analysis is the message. If more than one support type was provided in a message, the pre-dominant one was coded, in order to capture the primary intention and focus of message posters during message composition [28].
} 
Table 1. Factor loadings and cross-loadings (of reflective constructs)

\begin{tabular}{|l|c|c|c|}
\hline & Self-Disclosure (DIS) & Info. Support (INF) & Emo. Support (EMO) \\
\hline LIWC self-disclosure words in thread-init msgs & 0.93 & 0.15 & 0.02 \\
\hline LDA topical diversity of thread-init msgs & 0.80 & 0.10 & -0.01 \\
\hline Info. support count in thread responses & 0.11 & 0.97 & 0.48 \\
\hline Info. support length in thread responses & 0.17 & 0.96 & 0.37 \\
\hline Emo. support count in thread responses & -0.02 & 0.42 & 0.98 \\
\hline Emo. support length in thread responses & 0.03 & 0.45 & 0.97 \\
\hline
\end{tabular}

Table 2. Average Variance Extracted (AVE), Composite Reliability (CR), and latent variable correlations

\begin{tabular}{|l|c|c|c|c|c|c|c|}
\hline & AVE & CR & SLI & DIS & INF & EMO & HLP \\
\hline Subject Line Informativeness (SLI) & N/A & N/A & N/A & & & & \\
\hline Self-Disclosure (DIS) & 0.75 & 0.86 & 0.04 & $\mathbf{0 . 8 7}$ & & & \\
\hline Info. Support (INF) & 0.94 & 0.97 & 0.19 & 0.15 & $\mathbf{0 . 9 7}$ & & \\
\hline Emo. Support (EMO) & 0.95 & 0.97 & 0.15 & 0.01 & 0.44 & $\mathbf{0 . 9 7}$ & \\
\hline Thread Helpfulness (HLP) & N/A & N/A & 0.13 & 0.11 & 0.39 & 0.64 & N/A \\
\hline
\end{tabular}

Note: The diagonal elements are the square root of the AVE.

Machine (SVM) learning algorithm [29], to classify the remaining 53,277 messages into the two types of support messages automatically. The 10-fold crossvalidation method [30] was used to evaluate the trained program, yielding a $88.19 \%$ average classification accuracy. For each social support type, two reflective indicators were created - the total number of response messages belonging to the support type, and the length (total word counts) of those messages.

Helpfulness of discussion thread: To assess the helpfulness of a discussion thread, we incorporated two formative measures. First, we extracted the net vote value associated with each discussion thread as an indicator for thread helpfulness. Users of a reddit discussion forum can either vote like (upvote) or dislike (downvote) a discussion thread, specifying whether they found the thread helpful and relevant. The resulting net vote value associated with a thread is the difference between the number of upvotes and the number of downvotes for this thread. Since every forum member can upvote/downvote a thread, regardless of whether they posted in the thread or not, this indicator captures the helpfulness of a given discussion thread to forum users in general.

Second, for each thread, we used the number of follow-up messages posted by the thread initiator as the second formative indicator of overall thread helpfulness. Writing about one's own feelings and experiences associated one's own difficulties has been found to have therapeutic value, as it helps degrease negative emotions and alleviate loneliness, and it increases the sense of control over the difficulties [31]. Therefore, the more the thread initiator makes followup posts, the more helpful the thread can be to him/her. Additionally, follow-up posts made by thread initiators can be regarded as acknowledgements to support providers, signifying the helpfulness and relevance of these support messages. Therefore, this indicator captures the helpfulness of a given discussion thread to the thread initiator.

We chose to use Partial Least Squares (PLS) to test the model and hypotheses because PLS is appropriate for analyzing models that contain both formative and reflective indicators [32]. Additionally, the focus of PLS on predictive modeling aligns with the objective of our study. SmartPLS software package [33] was used for data analysis. ${ }^{2}$

\section{Results}

\subsection{Measurement Model Validation}

The first step of our analysis tested the adequacy of the measurement model. For reflective indicators, the indicator reliability (via indicator loadings, Table 1), convergent validity (via average variance extracted (AVE), Table 2), internal consistency reliability (via composite reliability (CR), Table 2), and discriminant validity (via cross loadings and the square root of

\footnotetext{
2 A bootstrapping procedure (500 resamples, as recommended in [34]) was used to assess the significance level of the hypothesized paths.
} 


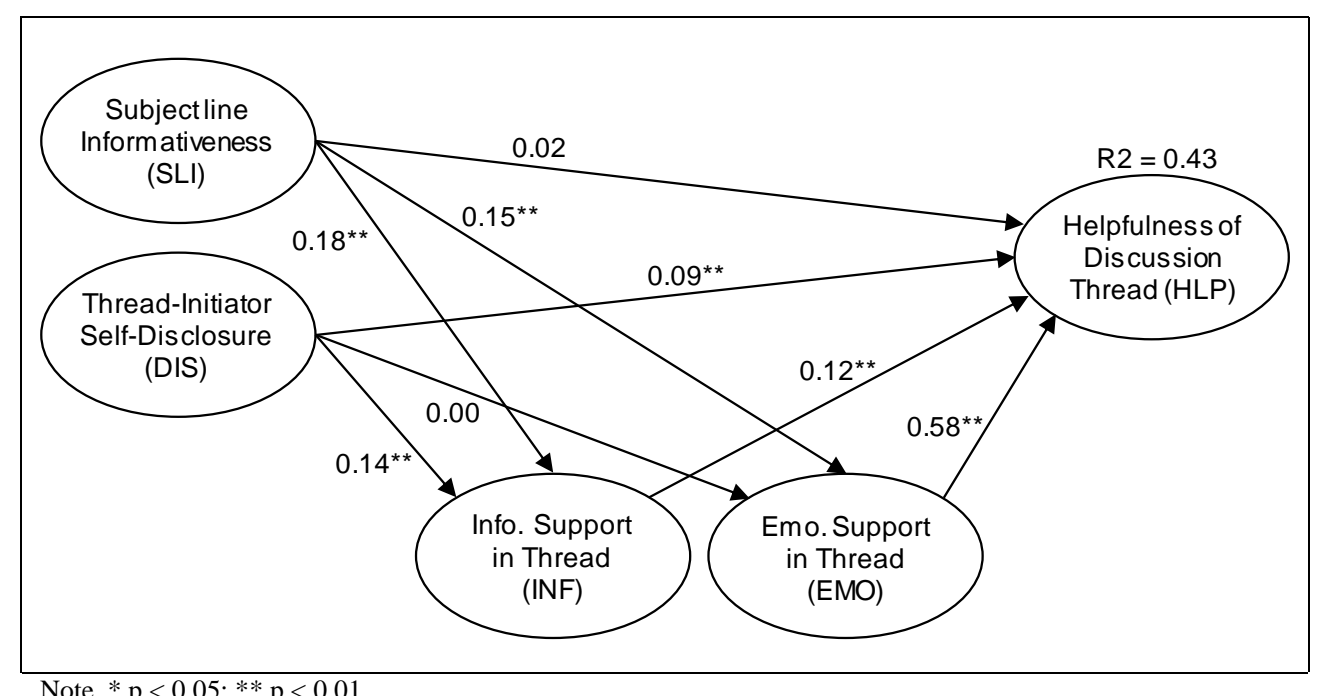

Figure 2. Result of PLS analysis

AVE, Tables 1 and 2) were assessed [35]. The results indicated that all the reflective constructs met the recommended threshold values. ${ }^{3}$ Regarding the formative construct (subject line informativeness and discussion thread helpfulness), we evaluated the construct validity (via indicator weights) and reliability (via multicollinearity test) [36]. The weights of the indicators for formative constructs are both significant at the 0.01 level, suggesting indicator validity. Multicollinearity was tested using VIF (variance inflator factor), resulting in values of 1.09 (formative indicators of title informativeness) and 1.06 (formative indicators of thread helpfulness), which are lower than the 3.3 threshold [37], suggesting the absence of multicollinearity.

\subsection{Structural Model Testing}

Table 3. Result of PLS analysis

\begin{tabular}{|c|c|l|c|}
\hline H1 & SLI $\rightarrow$ INF & $0.18^{* *}$ & Supported \\
\hline H2 & SLI $\rightarrow$ EMO & $0.15^{* *}$ & Supported \\
\hline H3 & SLI $\rightarrow$ HLP & 0.02 & Not Supported \\
\hline H4 & DIS $\rightarrow$ INF & $0.14 * *$ & Supported \\
\hline H5 & DIS $\rightarrow$ EMO & 0.00 & Not Supported \\
\hline H6 & DIS $\rightarrow$ HLP & $0.09 * *$ & Supported \\
\hline H7 & INF $\rightarrow$ HLP & $0.12 * *$ & Supported \\
\hline H8 & EMO $\rightarrow$ HLP & $0.58 * *$ & Supported \\
\hline
\end{tabular}

$* \mathrm{p}<0.05 ; * * \mathrm{p}<0.01$

${ }^{3}$ The generally accepted threshold for indicator loadings is 0.7 . The minimal values for acceptable AVE is 0.5 , and for CR is 0.7. The square roots of AVE should exceed the correlations of the correlations in corresponding rows and columns of Table 2 [35].
The result of the PLS test is shown in Figure 2. As can be seen in the figure, the proposed model explained $43 \%$ of the variance of the overall thread helpfulness. As for the hypotheses, thread subject-lineinformativeness successfully predicted both informational support received (SLI $\rightarrow \mathrm{INF}, \beta=0.18$, $P<0.01)$ and emotional support received (SLI $\rightarrow$ EMO, $\beta=0.15, P<0.01$ ) from support providers, supporting hypotheses $\mathrm{H} 1$ and $\mathrm{H} 2$. However, to the contrary of our hypothesis, subject line informativeness failed to predict thread helpfulness. Therefore, H3 was not supported.

With regard to the linguistic and content features of thread-initiating messages, self-disclosure in threadinitiating messages predicted informational support received from support providers (DIS $\rightarrow$ INF, $\beta=0.14$, $P<0.01)$ and the overall thread helpfulness (DIS $\rightarrow$ HLP, $\beta=0.09, P<0.01$ ), supporting H4 and H6. However, self-disclosure was not significantly related to emotional support. Thus, H5 was not supported.

Lastly, both informational and emotional support were positively associated with overall thread helpfulness, supporting $\mathrm{H} 7 \quad$ (INF $\rightarrow$ HLP, $\beta=0.12$, $P<0.01$ ) and H8 (EMO $\rightarrow$ HLP, $\beta=0.58, P<0.01$ ). Table 3 summarizes the findings of this study.

\section{Discussion and implications}

This study intends to discover factors that drive members of an online cannabis support forum to provide social support in discussion threads, and factors that lead to helpful discussion threads, through characterizing and testing the linguistic and content features of discussion thread subject lines and threadinitiating messages. Our findings highlight the idea that 
carefully formulated discussion thread subject lines and thread-initiating messages are capable of incentivizing members of online cannabis support forums to share useful informational and emotional support, leading to helpful discussion threads.

Specifically, thread subject-line informativeness is a significant predictor of the support seeker's receptions of informational and emotional support, as hypothesized. When initiating a thread, if the support seeker provides more information in the subject line, and/or mention about their withdrawal symptoms, the more likely forum users will click into the thread and provide social support. Our hypothesis about the relationship between thread subject-line informativeness and thread helpfulness was not supported, however. A possible explanation to this finding is that the positive relationship between subject-line informativeness and thread helpfulness is fully mediated by supportive responses in threads by other forum members. By applying Baron and Kenny's [38] procedure for testing mediation effects, we found that the relationship between subject-line informativeness and thread helpfulness in an unmediated model (without social support constructs) was positive and significant $(\beta=0.14, P<0.01)$. After introducing the informational and emotional support constructs to the model, their relationship changed to non-significant. This supports our explanation, that informational and emotional support fully mediated the relationship between subject-line informativeness and thread helpfulness.

As for the factors involving the characteristics of thread-initiating messages, our study highlights the importance of support seekers' self-disclosure in messages, as our findings shows that self-disclosure in thread-initiating messages is positively associated with informational support and thread helpfulness. However, the relationship between self-disclosure in thread-initiating messages and emotional support responses in threads was not significant. It implies that regardless of the levels of support seekers' selfdisclosure in thread-initiating messages, support providers tend to provide emotional support in threads. This is the case in online support forums where a supportive norm can be developed [39], driving forum members to provide welcoming messages, encouragements, acknowledgements, and so on emotional support - to support seekers. Future works can look more into this phenomenon in online cannabis support forums.

Lastly, forum users' participations in discussion threads to offer informational and emotional support are critical to thread helpfulness, as hypothesized. The strong relationship between emotional support and thread helpfulness suggests the particular importance of providing emotional support in the context of online cannabis support forums, whose users generally experience intense cannabis withdrawal symptoms during the first few weeks of sobriety [17] and thus are in need of emotional uplift.

Our study provides implications to research and practice. First, while there is an increasing number of people joining online cannabis support forums for exchanging support, little is known about the social and behavioral dynamics of this forum type. Our study provides insights into the dynamics of an online cannabis support forum, which lead to more future research opportunities regarding the behaviors of support seekers and providers, and their interactions in threads, in order to identify ways of helping cannabis quitters.

Additionally, the use of automated content analysis methods in this study allows us to look into linguistic and content features of discussion messages. Our findings suggest that the content of thread subject lines and thread-initiating messages can have impacts on thread activities and thread helpfulness. Future work can investigate more into the various linguistic patterns of different messages in a thread as well as their effects. Automated methods also allow us to analyze a large volume of online user-generated content, which suggests useful research tools for researchers attempting to conduct online studies involving large amount of text content.

Our findings can also be used as guidance for forum users seeking for support from others, as they highlight the ways of composing thread-initiating messages and subject lines for promoting social support exchange and helpful discussions. For forum designers, providing forum features that allow and supports displaying long thread subject lines, and highlights context-related information (e.g., emotional and physical-behavioral symptoms in the case of online cannabis support forum) in subject lines can be conducive to thread vibrancy. For forum administrators and healthcare professionals, it is important to encourage the provisions of social support, especially emotional support, from forum users. This helps anxious support seekers feel that they are supported and are not fighting addiction alone, leading to increased follow up postings from support seekers and overall thread helpfulness to forum users.

\section{Limitations and Conclusion}

This study has some limitations that could be addressed in future research. There are some inherent limitations associated with content analysis. In this study we used the "message" as the unit of analysis and classified each message response into either 
informational or emotional support. However, it is likely that both types of social support can be provided equally within a message. As a result, detailed information regarding the existence of, and the degree to which, different support types within each message is missing in our study. Additionally, a limitation of conducting automated content analysis using machine learning techniques is the introduction of prediction errors. To compensate for these problems, future studies can employ a mixed-method methodology, consisting automated content analysis and other methods such as survey questionnaires or ethnographic observations, to triangulate the findings.

In spite of these limitations, we believe that by investigating the social interaction behaviors of online cannabis forum users, this study provides useful insights for both researchers and practitioners. As the number of people attempting to quit cannabis increases, we hope this study will inspire helpful interventions benefiting those people.

\section{Acknowledgement}

This work was supported by the Institute of Cannabis Research (ICR) at Colorado State University - Pueblo.

\section{References}

[1] A. Geiger, "About Six-in-Ten Americans Support Marijuana Legalization," Pew Research Center, 2008, available at http://www.pewresearch.org/facttank/2018/01/05/americans-support-marijuana-legalization/

[2] M. Cerdá, M. Wall, T. Feng, K. M. Keyes, A. Sarvet, J. Schulenberg, P. M. O’Malley, R. L. Pacula, S. Galea, D. S. Hasin, "Association of State Recreational Marijuana Laws with Adolescent Marijuana Use," JAMA Pediatrics, vol. 171, no. 2, 2017, pp. 142-149.

[3] C. Hopfer, "2.2 Clinical Issues Arising in the Context of Adult Recreational Marijuana Legalization," Journal of the American Academy of Child \& Adolescent Psychiatry, vol. 56, no. 10,2017 , pp. S4.

[4] J. R. Hughes, S. Naud, A. J. Budney, J. R. Fingar, and P. W. Callas, "Attempts to Stop or Reduce Daily Cannabis Use: An Intensive Natural History Study," Psychology of Addictive Behaviors, vol. 30, no. 3, 2016, pp. 389-397.

[5] M. R. Pearson, B. S. Liese, R. D. Dvorak, and Marijuana Outcomes Study Team, "College Student Marijuana Involvement: Perceptions, Use, and Consequences across 11 College Campuses," Addictive Behaviors, vol. 66, 2017, pp. 83-89.
[6] K. P. Hill, "Cannabis Use and Risk for Substance Use Disorders and Mood or Anxiety Disorders," JAMA, vol. 317, no. 10, 2016, pp. 1070-1071.

[7] C. Greiner, A. Chatton, and Y. Khazaal, "Online SelfHelp Forums on Cannabis: A Content Assessment," Patient Education and Counseling, vol. 100, 2017, pp. 1943-1950.

[8] K. B. Wright and S.B. Bell, "Health-Related Support Groups on the Internet: Linking Empirical Findings to Social Support and Computer-Mediated Communication Theory," Journal of Health Psychology, vol. 8, no. 1, 2003, pp. 39-54.

[9] U. Pfeil, "Online Support Communities," in Social Computing and Virtual Communities, P. Zaphiris, and C. S. Ang (Eds.), Chapman \& Hall, 2009, pp. 121-150.

[10] S. J. Sowles, M. J. Krauss, L. Gebremedhn, and P. A. Cavazos-Rehg, "“I Feel Like I've Hit the Bottom and Have No Idea What to Do": Supportive Social Networking on Reddit for Individuals with a Desire to Quit Cannabis Use," Substance Abuse, vol. 38, no. 4, 2017, pp. 477-482.

[11] T. P. Ellingstad, L. C. Sobell, M. B. Sobell, L. Eickleberry, and C. J. Golden, "Self-Change: A Pathway to Cannabis Abuse Resolution," Addictive Behaviors, vol. 31, no. 3, 2006, pp. 519-530.

[12] X. Luo, J. Zhang, and W. Duan, "Social Media and Firm Equity Value," Information Systems Research, vol. 24, no. 1, 2013, pp. 146-163.

[13] J. W. Pennebaker, M. R. Mehl, and K. G. Niederhoffer, "Psychological Aspects of Natural Language Use: Our Words, Ourselves," Annual Review of Psychology, vol. 24, no. 1, 2003, pp. 547-577.

[14] L. K. Suzuki and J. P. Calzo, "The Search for Peer Advice in Cyberspace: An Examination of Online Teen Bulletin Boards about Health and Sexuality," Applied Developmental Psychology, vol. 25, 2004, pp. 685-698.

[15] J. Skogs, "Subject Line Preferences and Other Factors Contributing to Coherence and Interaction in Student Discussion Forums," Computers \& Education, vol. 60, no. 1, 2013, pp. 172-183.

[16] S. M. Mudambi and D. Schuff, "Research Note: What Makes a Helpful Online Review? A Study of Customer Reviews on Amazon.com," MIS Quarterly, vol. 34, no. 1, pp. 185-200.

[17] D. J. Allsop, M. M. Norberg, J. Copeland, S. Fu, and A. J. Budney, "The Cannabis Withdrawal Scale Development: Patterns and Predictors of Cannabis Withdrawal and Distress," Drug and Alcohol Dependence, vol. 119, 2011, pp. 123-129.

[18] L. C. Jiang, N. N. Bazarova, and J. T. Hancock, "The Disclosure-Intimacy Link in Computer-Mediated Communication: An Attributional Extension of the 
Hyperpersonal Model," Human Communication Research, vol. 37 , no. 1,2011 , pp. 58-77.

[19] A. Z. Y. Barak and O. Gluck-Ofri "Degree and Reciprocity of Self-Disclosure in Online Forums," CyberPsychology \& Behavior, vol. 10, no. 3, 2007. pp. 407417.

[20] K. M. Barnes and S. Duck "Everyday Communicative Contexts for Social Support," in Communication of Social Support: Messages, Interactions, Relationships, and Community, B. R. Burleson, T. L. Albrecht, and I. G. Sarason (Eds.), Thousand Oaks, CA: Sage Publications, 1994, pp. 175-194.

[21] J. Håkansson and H. Montgomery, "Empathy as an Interpersonal Phenomenon," Journal of Social and Personal Relationships, vol. 20, no. 3, 2003, pp. 267-284.

[22] B. Nonnecke and J. Preece, "Silent Participants: Getting to Know Lurkers Better," in From Usenet to CoWebs: Interacting with Social Information Spaces, C. Leug and D. Fisher (Eds.), Amsterdam, Holland: Springer-Verlag, 2003.

[23] V. J. Duriau, R. K. Reger, and M. D. Pfarrer, "A Content Analysis of the Content Analysis Literature in Organization Studies," Organizational Research Methods, vol. 10, no. 1, 2007, pp. 5-34.

[24] D. E. Callaghan, M. G. Graff, and J. Davies "Revealing All: Self-Disclosure Rates in Laboratory-Based Online Research," Cyberpsychology, Behavior, and Social Networking, vol. 16, no. 9, 2013, pp. 690-694.

[25] L. Wu, "Social Network Effects on Productivity and Job Security: Evidence from the Adoption of a Social Networking Tool," Information Systems Research, vol 24, no. 1, 2013, pp. 30-51.

[26] D. M. Blei, "Probabilistic Topic Models," Communications of the ACM, vol. 55, no. 4, 2012, pp. 77-84.

[27] K.-Y. Huang, P. Nambisan, and Ö. Uzuner, "Informational Support or Emotional Support: Preliminary Study of an Automated Approach to Analyze Online Support Community Contents," in Proceedings of the International Conference on Information Systems (ICIS'10), St. Louis, MO, 2010.

[28] J. Finn, "An Exploration of Helping Processes in an Online Self-Help Group Focusing on Issues of Disability," Health \& Social Work, vol. 24, no. 3, 1999, pp. 220-231.

[29] T. Joachims, T. "Text Categorization with Support Vector Machines: Learning with Many Relevant Features," in Proceedings of the European Conference on Machine Learning (ECML'98), Chemnitz, Germany, 1998, pp. 137142.

[30] F. Sebastiani, "Machine Learning in Automated Text Categorization," ACM Computing Surveys, vol. 34, no. 1, 2002, pp. 1- 47.

[31] A. Barak, M. Boniel-Nissim, and J. Suler, "Fostering Empowerment in Online Support Groups," Computers in Human Behavior, vol. 24, no. 5, 2008, pp. 1867-1883.

[32] W. W. Chin and P. R. Newsted "Structural Equation Modeling Analysis with Small Samples Using Partial Least Squares," in Statistical Strategies for Small Sample Research, R. Hoyle (Ed.), Thousand Oaks, CA: Sage Publications, 1999, pp. 307-341.

[33] C. M. Ringle, S. Wende, and A. Will, SmartPLS. Hamburg, Germany, 2005, available at http://www.smartpls.de.

[34] C. Z. Mooney and R. D. Duval, Bootstrapping: A Nonparametric Approach to Statistical Inference, Newbury Park, CA: Sage, 1993.

[35] C. Fornell and D. F. Larcker "Evaluating structural equation models with unobservable variables and measurement error," Journal of Marketing Research, vol. 18, 1981, pp. 39-50.

[36] W. W. Chin, "The Partial Least Squares Approach to Structural Equation Modeling," in Modern Methods for Business Research, G. A. Marcoulides (Ed.), London: Psychology Press, 1998, pp. 295-336.

[37] S. Petter, D. Straub, and A. Rai, "Specifying Formative Constructs in Information Systems Research," MIS Quarterly, vol. 31, no. 4, 2007, pp. 623-656.

[38] R. M. Baron and D. A. Kenny, "The ModeratorMediator Variable Distinction in Social Psychological Research: Conceptual Strategic, and Statistical Considerations," Journal of Personality and Social Psychology, vol. 51, no. 6, 1986, pp. 1173-1182.

[39] D. Maloney-Krichmar and J. Preece, "The Meaning of An Online Health Community in the Lives of its Members: Roles, Relationships and Group Dynamics," in 2002 International Symposium on Technology and Society (ISTAS'02), Raleigh, NC, 2002, pp. 20-27. 